\title{
Versatile Method to Expand the Morphology Library of Block Copolymer Solution Self-Assemblies with Tubular Structures
}

Xiao-Li Sun, Dong-Ming Liu, Shuai Pei, Kang-Kang Li, Wen-Ming Wan *

State Key Laboratory of Heavy Oil Processing and Centre for Bioengineering and Biotechnology, China University of Petroleum (East China), 66 Changjiang West Road, Qingdao Economic Development Zone, Qingdao, Shandong, 266580, People's Republic of China.

Correspondence and requests for materials should be addressed to wanwenming@upc.edu.cn 


\section{Experimental}

Materials. The azobisisobutyronitrile (AIBN) initiator was recrystallized in methanol. Styrene was purified by passing it through a neutral alumina column, followed by distillation under reduced pressure. S-1-dodecyl-S-( $\alpha, \alpha^{\prime}$-dimethyl- $\alpha$ ' -acetic acid) trithiocarbonate (TC) and poly(4-vinylpyridine) $(\mathrm{P} 4 \mathrm{VP}-\mathrm{TC})$ was reported previously $\left(\mathrm{M}_{\mathrm{n}, \mathrm{NMR}}=10400 \mathrm{~g} \mathrm{~mol}^{-1}, \mathrm{DP}_{\mathrm{NMR}}=96, \bigoplus_{\mathrm{GPC}}=1.08\right) .{ }^{1}$ All other solvents and chemicals were used without further purification.

\section{Characterizations}

Nuclear Magnetic Resonance (NMR) Spectra. The ${ }^{1} \mathrm{H}$ NMR measurements were performed on Bruker DMX300 spectrometer in $\mathrm{CDCl}_{3}$ using tetramethylsilane as an internal reference.

Gel permeation chromatography (GPC). The molecular weight and molecular weight distribution were determined on a Waters 150C gel permeation chromatography (GPC) equipped with three ultrastyragel columns $\left(500,10^{3}, 10^{4} \AA\right)$ in series and RI 2414 detector at $30{ }^{\circ} \mathrm{C}$, and THF was used as eluent at a flow rate of $1.0 \mathrm{~mL} / \mathrm{min}$. Monodispersed polystyrene standards were used in the calibration of molecular weight and molecular weight distribution.

Field emission scanning electron microscopy (FESEM). The FESEM images were measured on a JEOL JSM-6700F. The samples were prepared by placing a drop of the polymer solution in methanol on copper grids, and gilding a shell of Pt nano-particle.

Transmission Electron Microscopy. The transmission electron microscope (TEM) observations were performed on a Hitachi H-800 TEM at an accelerating voltage of $200 \mathrm{kV}$. The samples for TEM observations were prepared by depositing a drop of the polymer solution in methanol on copper grids.

\section{Methods}

\section{Synthesis of P4VP-b-PSt block copolymers with highly asymmetric composition and high} molecular weight. S-1-dodecyl-S-( $\alpha, \alpha^{\prime}$-dimethyl- $\alpha$ "-acetic acid) trithiocarbonate (TC) and poly(4-vinylpyridine) $(\mathrm{P} 4 \mathrm{VP}-\mathrm{TC})$ was reported previously $\left(\mathrm{M}_{\mathrm{n}, \mathrm{NMR}}=10400 \mathrm{~g} \mathrm{~mol}^{-1}, \bigoplus_{\mathrm{GPC}}=1.08\right) .{ }^{1} \mathrm{~A}$ typical procedure is as follows. The P4VP-TC (0.104 g, $0.01 \mathrm{mmol})$, St (1.04 g, $10 \mathrm{mmol})$, AIBN (0.2 
$\mathrm{mg}, 20 \mu \mathrm{L}$ of freshly prepared $10 \mathrm{mg} / \mathrm{mL}$ AIBN stock solution in methanol, $1 \mu \mathrm{mol})$ and methanol (1.0 $\mathrm{mL}$ ) were added into a $5 \mathrm{~mL}$ glass polymerization tube with a magnetic bar, followed by three freeze-pump-thaw cycles. After being sealed under vacuum, the sealed polymerization tube was placed in an oil bath at $80^{\circ} \mathrm{C}$. The polymerization was quenched in ice-water. Polymer was precipitated by pouring the polymer solution into excess petroleum ether while stirring. Polymer powder was collected by filtration, and then dried in a vacuum oven at room temperature overnight. $0.82 \mathrm{~g}$ of the product was obtained, with conversion of $68 \%$.

Self-assembly of P4VP-b-PSt block copolymers in methanol. Block copolymer was dissolved in cosolvent THF firstly to reach a concentration at $10 \mathrm{mg} / \mathrm{mL}$. Then, $9.0 \mathrm{~mL}$ selective solvent methanol was added dropwise to $1.0 \mathrm{~mL}$ polymer/THF solution by using syringe pump at a rate of $1.0 \mathrm{~mL} / \mathrm{hour}$. The morphologies of assemblies for TEM observation were frozen by pouring assemblies solution into excessive methanol (ten folds) under vigorous stirring, according to literatures. ${ }^{2}$. Then samples were deposited on copper grids immediately after sample preparation.

Molecular dynamics (MD) simulations. Coarse-grained MD simulations on P4VP- $b$-PS BCPs were performed with same asymmetry (1: 10, 2: 20 and 4: 40) by the Gromacs 4.5.5 package. ${ }^{3}$ According to the Martini Force Field, ${ }^{4}$ the coarse grained model of P4VP-b-PS was built as follows: Each repeating unit of the backbone $\left(\mathrm{CH}_{2}-\mathrm{CH}\right)$ was represented by $\mathrm{C} 1$ bead, phenyl and pyridyl moieties were represented by SC4 and Nd beads, and solvent was represented by P3 bead. The simulations of the copolymer system were accomplished in $1 \mu$ s to reach the equilibrium state in a cubic box with periodic boundary conditions in three directions. The side length of the box was $20 \mathrm{~nm}$. The canonical ensemble NPT was performed at $298 \mathrm{~K}$ and 1 atm for each system, and the integration step was set as $20 \mathrm{fs}$. The temperature coupling was set by the Berendsen thermostat, and the pressure coupling was set by the Berendsen barostat. ${ }^{5}$ The cut-off for non-bonded interactions was set at $1.2 \mathrm{~nm}$ with the standard shift functions in Gromacs, where the van der Waals interaction was shifted from 0.9 to $1.2 \mathrm{~nm}$, and the electrostatic interaction was shifted from 0 to $1.2 \mathrm{~nm}$. 
Radial distribution functions (RDFs) discussion. For a better understanding of how molecular weights affect the resulting morphology, the radial distribution functions of methanol solvent around each repeating unit of P4VP- $b$-PS BCPs were calculated, as shown in Figure S5, where VP, St and x refer to 4-vinylpyridine, styrene and the ordinal number of 4-vinylpyridine and styrene counting from P4VP head of P4VP- $b$-PS BCPs, respectively. The distribution probability of methanol solvent around the first $4 \mathrm{VP}$ unit of $\mathrm{P} \mathrm{VP}_{1}-b-\mathrm{PS}_{10}$ is 1.08 , higher than 1 , while that for the first St unit is 0.53 , much lower than 1, indicating the first 4VP unit is well dissolved in methanol as shell and the first St unit precipitates from methanol as core. This simulation result is totally consistent with the fact that methanol solvent is good solvent for P4VP block and poor solvent for PS block of BCPs. And the neighboring two repeating units with the greatest difference of methanol distribution probability are therefore used to determine the core/shell interface of particle, as pointed out by two arrows in Figure S5. So the core/shell interfaces are between VP1-St1, VP2-St1 and VP4-St1 for P4VP $1-b$-PS 10 ,

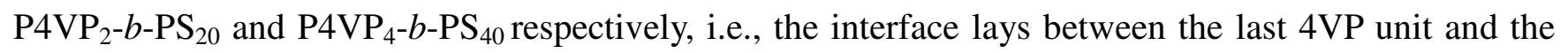
first St unit. Further reveal of the effect of molecular weight on final morphologies were carried out by comparing the RDFs of methanol solvent around the core/shell interfaces, as shown in Figure S6. As molecular weight of BCPs increasing, the distribution probability of methanol solvent around the last 4VP unit decreases from 1.09 to 1.08 and further to 0.91 (lower than 1), indicating that part of the last 4VP unit was pulled beneath the core/shell interface by core-forming PS block. Meanwhile, as molecular weight of BCPs increasing, the distribution probability of methanol around the first St unit decreases as well, from 0.53 to 0.44 and further to 0.34 , indicating that methanol was excluded from the core of particles. The molecular weight therefore plays important roles in the location of core/shell interface, the distribution of selective solvent in the core, which in return affect the final morphologies from spherical micelle to ellipsoid-like micelle and bi-tubular structure.

\section{References.}

(1) (a) Wan, W. M.; Pan, C. Y., Macromolecules 2010, 43, 2672-2675; (b) Wan, W. M.; Pan, C. Y., Macromolecules 2008, 41, 5085-5088; (c) Wan, W. M.; Pan, C. Y., Chem. Commun. 2008, 43, 
5639-5641; (d) Wan, W. M.; Pickett, P. D.; Savin, D. A.; McCormick, C. L., Polym. Chem. 2014, 5, 819-827; (e) Wan, W. M.; Cheng, F.; Jäkle, F., Angew. Chem. Int. Ed. 2014, 53, 8934-8938.

(2) (a) Luo, L.; Eisenberg, A., Langmuir 2001, 17, 6804-6811; (b) Liu; Wu, J.; Kim, J. S.; Eisenberg, A., Langmuir 2006, 22, 419-424; (c) Vyhnalkova, R.; Eisenberg, A.; van de Ven, T. G. M., J. Phys. Chem. B 2008, $112,8477-8485$.

(3) Hess, B.; Kutzner, C.; van der Spoel, D.; Lindahl, E., J. Chem. Theory Comput. 2008, 4, 435-447.

(4) Marrink, S. J.; Risselada, H. J.; Yefimov, S.; Tieleman, D. P.; de Vries, A. H., J. Phys. Chem. B 2007, $111,7812-7824$.

(5) Berendsen, H. J. C.; Postma, J. P. M.; van Gunsteren, W. F.; DiNola, A.; Haak, J. R., J. Chem. Phys. 1984, $81,3684-3690$. 


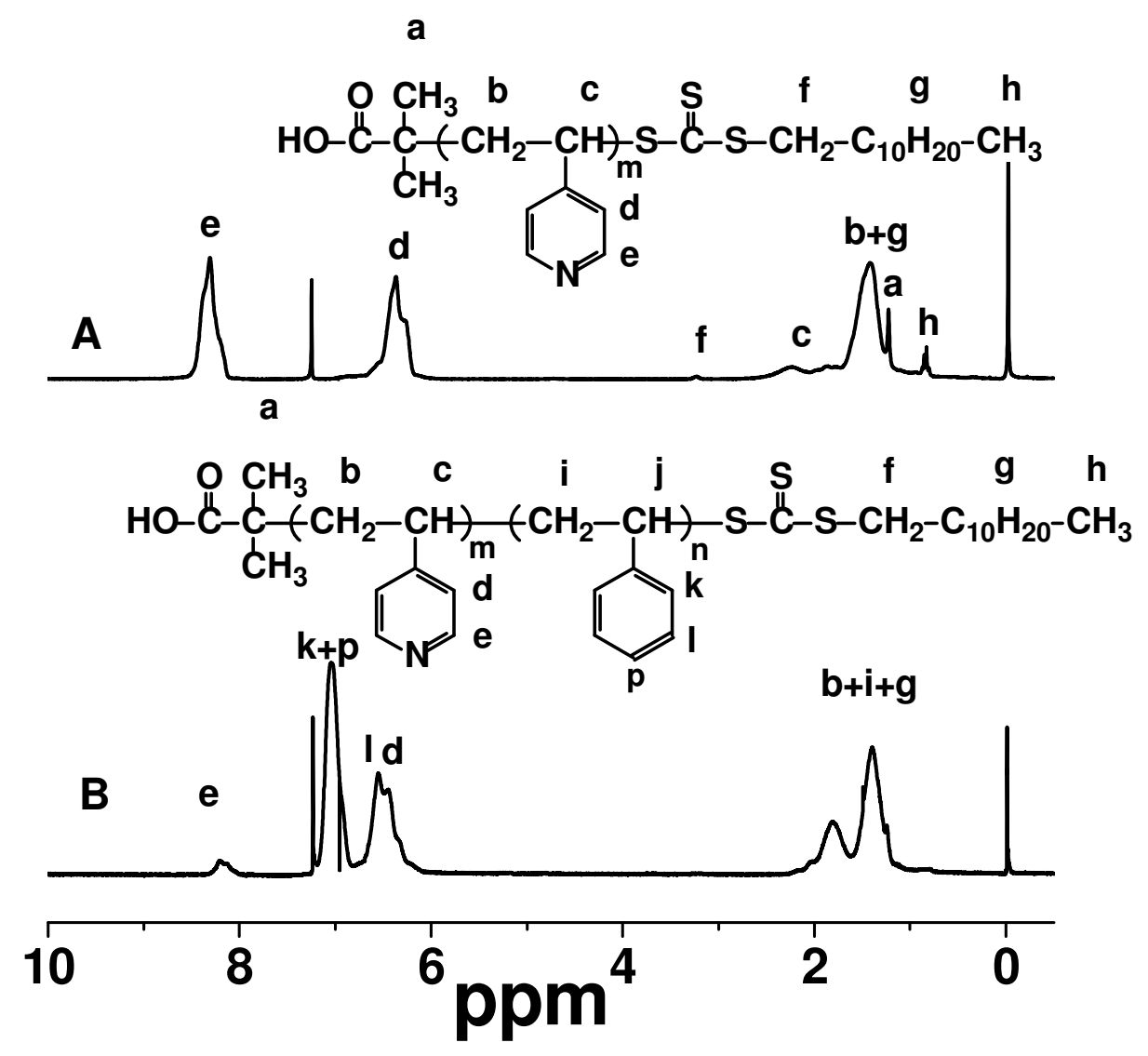

Figure S1. Representative ${ }^{1} \mathrm{H}$ NMR spectra of A) TC-terminated poly(4-vinylpyridine) (P4VP-TC); B) P4VP- $b$-PS block copolymer prepared by RAFT PISR of styrene (St) with P4VP-TC/St $(1.04 \mathrm{~g}) / \mathrm{AIBN}=$ $10 / 20000 / 1$ in methanol $(1.0 \mathrm{~mL})$ at $80^{\circ} \mathrm{C}$ for $24 \mathrm{~h}$. 


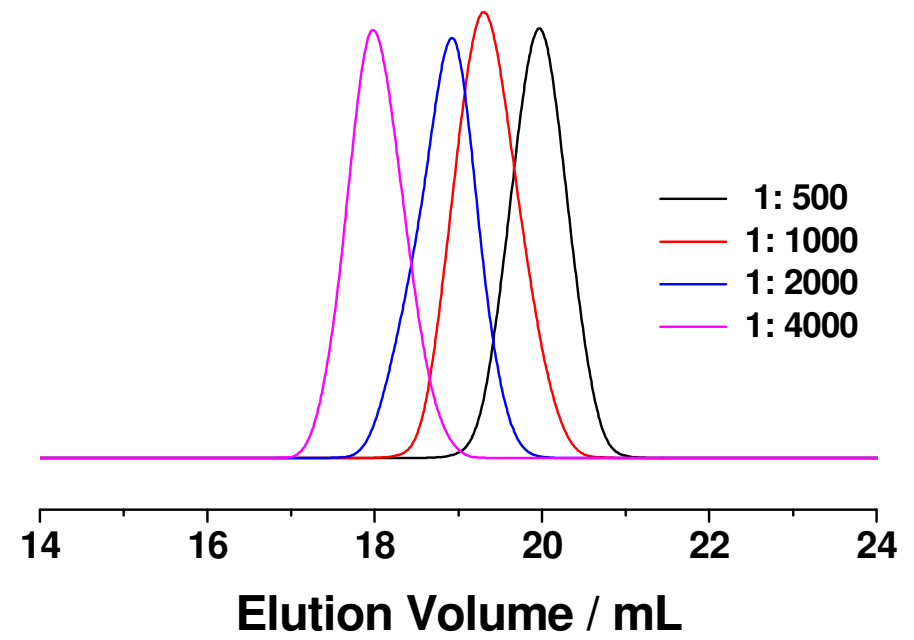

Figure S2. GPC curves of P4VP- $b$-PS block copolymers prepared by RAFT PISR of styrene (St) with $\mathrm{P} 4 \mathrm{VP}-\mathrm{TC} / \mathrm{St}(1.04 \mathrm{~g})=1: \mathrm{X}$ in methanol $(1.0 \mathrm{~mL})$ at $80^{\circ} \mathrm{C}$ for $24 \mathrm{~h}$. 
Table S1. Data for P4VP-b-PS via RAFT PISR and morphologies obtained via BCP self-assembly in methanol.

\begin{tabular}{ccccccccc}
\hline No. & Feed ratio & Conversion (\%) & $\mathrm{DP}_{\mathrm{P}_{4} \mathrm{VP}}$ & $\mathrm{DP}_{\mathrm{PS}}$ & $\mathrm{P}_{4} \mathrm{VP} \%^{\mathrm{a}}$ & $\mathrm{M}_{\mathrm{n}, \mathrm{NMR}}(\mathrm{kDa})^{\mathrm{a}}$ & $\mathrm{M}_{\mathrm{w}} / \mathrm{M}_{\mathrm{n}}{ }^{\mathrm{b}}$ & Morphology \\
\hline 1 & $1: 500$ & 71 & 96 & 341 & $22.0 \%$ & 45.9 & 1.22 & Spherical micelles \\
2 & $1: 1000$ & 68 & 96 & 663 & $12.6 \%$ & 79.4 & 1.25 & vesicles \\
3 & $1: 2000$ & 58 & 96 & 1112 & $7.95 \%$ & 126.1 & 1.24 & tubular bead dumbbells \\
4 & $1: 4000$ & 48 & 96 & 1834 & $4.97 \%$ & $\mathbf{2 0 1 . 2}$ & 1.25 & tubules \\
\hline
\end{tabular}

a Calculated from the ${ }^{1} \mathrm{H}$ NMR spectroscopy. ${ }^{\mathrm{b}}$ Measured by GPC. 


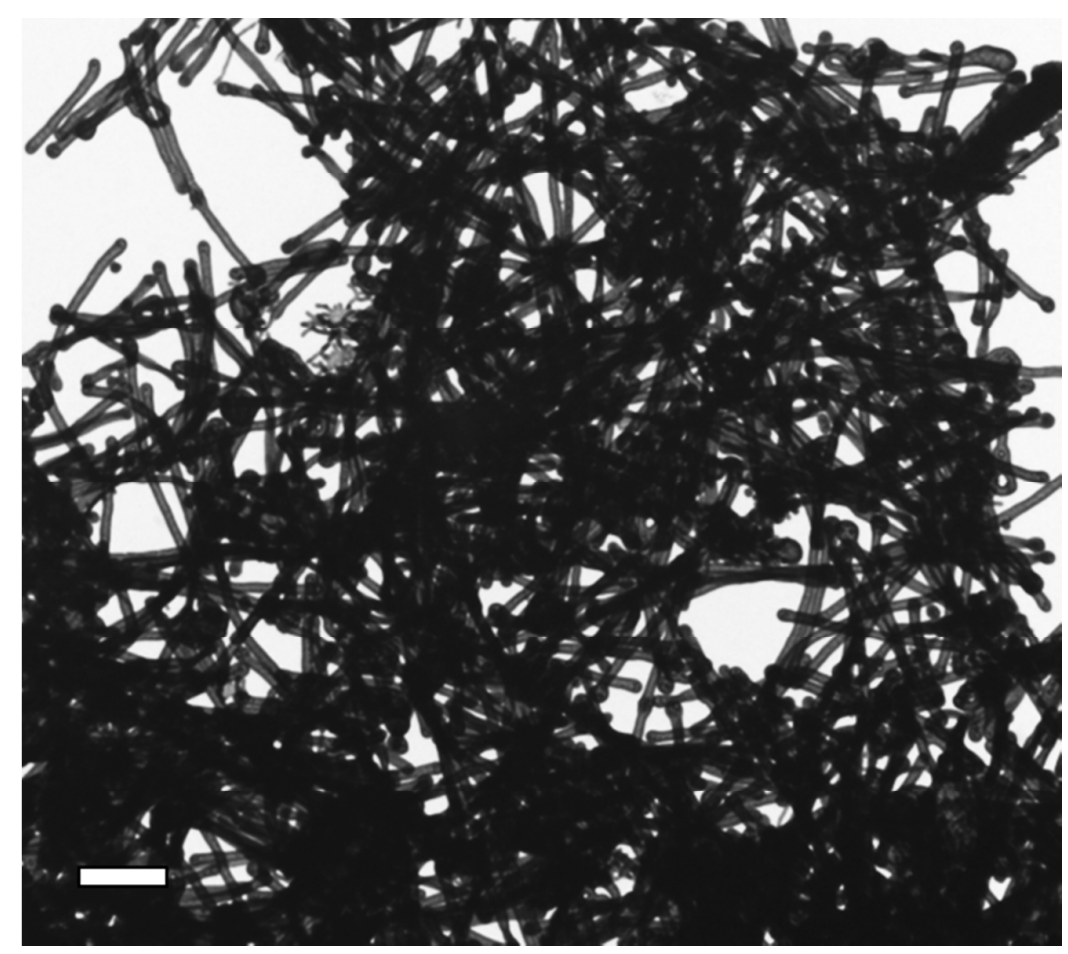

Figure S3. Tubules of $\mathrm{P}_{4} \mathrm{VP}_{96}-b-\mathrm{PS}_{1834}$ aggregates formed by BCP self-assembly in methanol. Starting BCP concentration is $10 \mathrm{mg} / \mathrm{mL}$. Scale bar is $2 \mu \mathrm{m}$. 


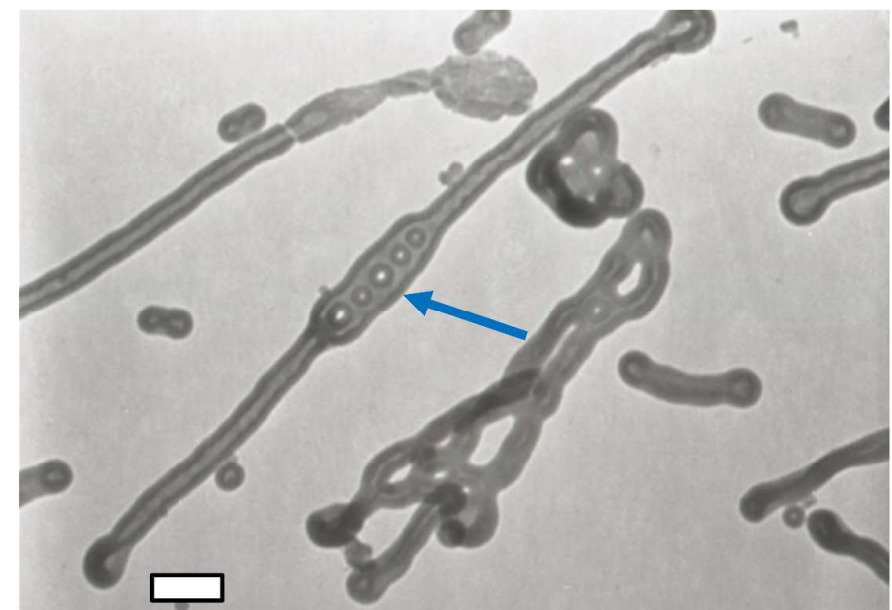

Figure S4. Tubular structure with fused vesicles of $\mathrm{P} 4 \mathrm{VP}_{96}-b-\mathrm{PS}_{1834}$ aggregates formed by BCP self-assembly in methanol. Starting BCP concentration is $10 \mathrm{mg} / \mathrm{mL}$. Scale bar is $2 \mu \mathrm{m}$. 

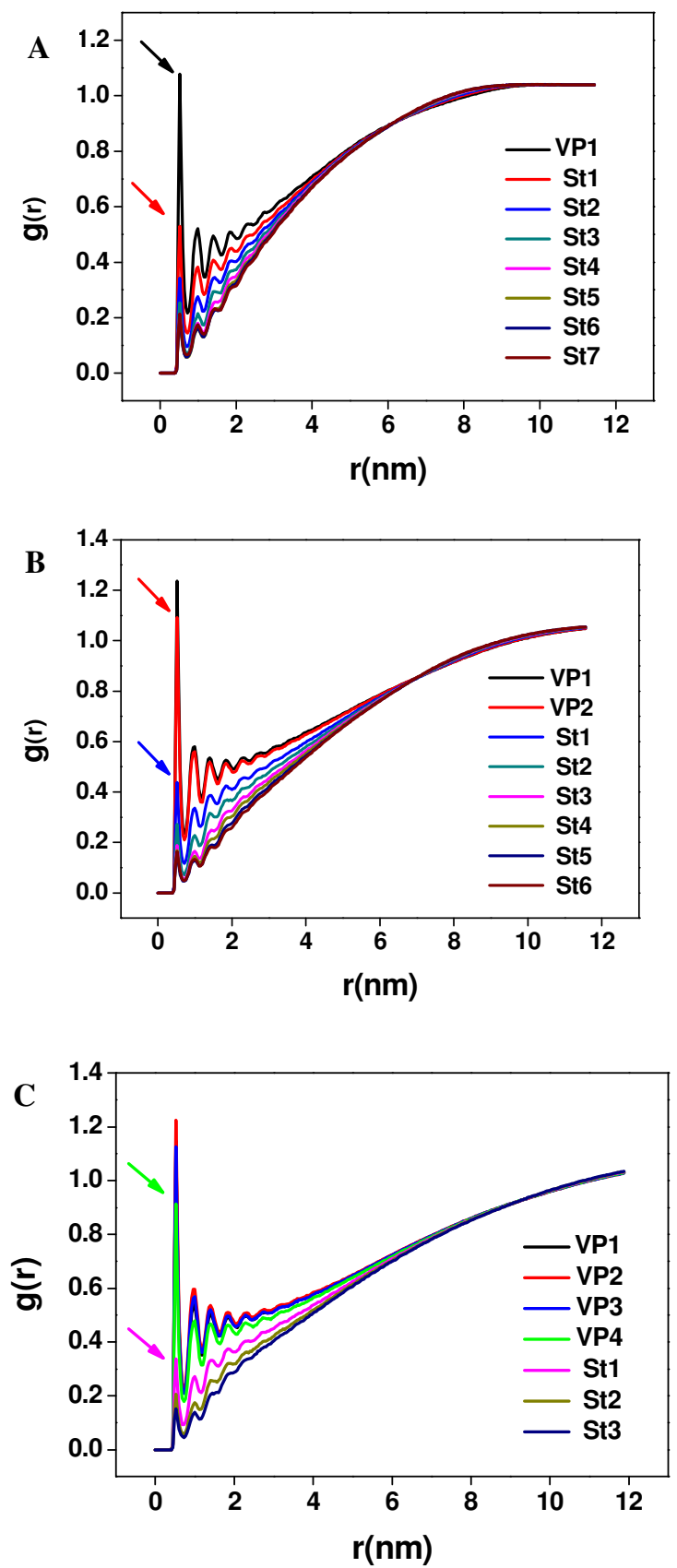

Figure S5. Radial distribution functions (RDFs) of methanol solvent around VPx and

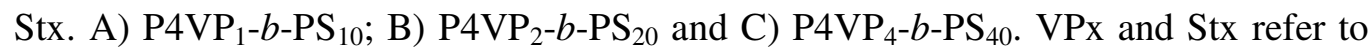
the ordinal number of 4-vinylpyridine and styrene counting from $\mathrm{P} 4 \mathrm{VP}$ head of P4VP- $b$-PS block copolymers, respectively. The neighboring two repeating units with the greatest difference of methanol distribution probability are pointed out by two arrows, where the core/shell interfaces of particle are determined. 

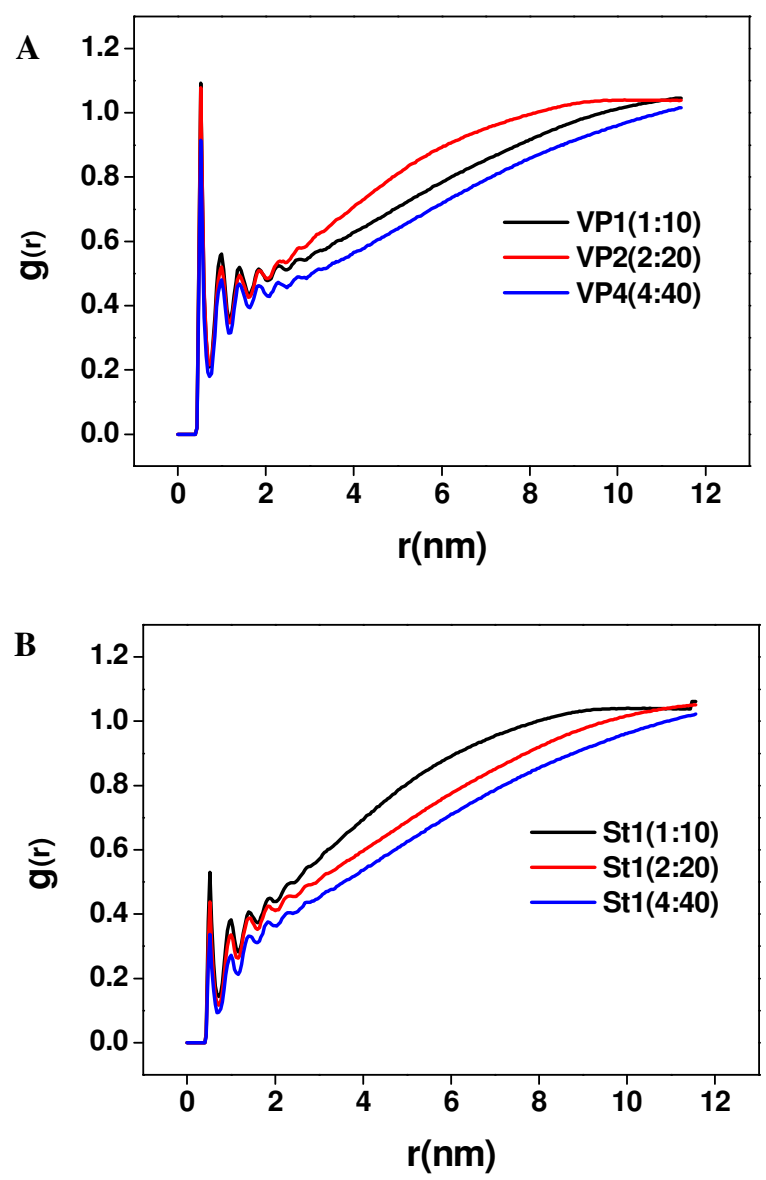

Figure S6. Radial distribution functions (RDFs) of methanol solvent around the core/shell interfaces, i.e., the last 4VP unit (A) and the first St unit (B) of P4VP- $b$-PS block copolymers. 